\title{
Civilisations
}

Revue internationale d'anthropologie et de sciences

humaines

$63 \mid 2014$

L'Ancestralité revisitée

\section{Sirène, rois et démocratie}

Ancestralisation et conscience nationale dans un rituel inédit (2001, Sud de Madagascar)

\section{Thomas Mouzard}

\section{OpenEdition}

\section{Journals}

Édition électronique

URL : http://journals.openedition.org/civilisations/3668

DOI : 10.4000/civilisations.3668

ISSN : 2032-0442

\section{Éditeur}

Institut de sociologie de l'Université Libre de Bruxelles

\section{Édition imprimée}

Date de publication : 30 septembre 2014

Pagination : 45-61

ISSN : 0009-8140

\section{Référence électronique}

Thomas Mouzard, « Sirène, rois et démocratie », Civilisations [En ligne], 63 | 2014, mis en ligne le 30 septembre 2018, consulté le 20 avril 2019. URL : http://journals.openedition.org/civilisations/3668 DOI : 10.4000/civilisations.3668 


\title{
Ancestralisation et conscience nationale dans un rituel inédit (2001, Sud de Madagascar)
}

\author{
Thomas MOUZARD
}

Résumé : En 2001, alors que Madagascar traverse une nouvelle crise démocratique, un petit cercueil traverse le Sud du pays d'est en ouest. Des centaines de communautés villageoises sont reliées par cette trajectoire qui emprunte des sillons historiques du territoire national. Au cours de ce relais funéraire de trois mois, l'identité du razamasy, "ancêtre-sacré " en circulation va changer à plusieurs reprises : d'abord lémurien il terminera sirène. On assiste ainsi à la production d'une ancestralité, qui doit assurer de sa pertinence. La problématique retenue ici est celle de la légitimation, fondée sur des logiques anciennes et toujours prégnantes du pouvoir, qui semble réactivée par une conjoncture électorale. Cet article met finalement l'accent sur la manifestation d'une conscience nationale à travers ce phénomène d'ancestralisation.

Mots-clés : ancestralité, rituel, politique, parenté, nation, démocratie, royauté, Madagascar, sirène.

\begin{abstract}
In 2001, while Madagascar went through a new democratic crisis, a small coffin crosses the South of the country from east to west. Hundreds of village communities are connected by this trajectory which follows historic furrow of the national territory. During this funeral relay of three months, the identity of razamasy, the "sacred ancestor" has changed several times: first lemur he finished mermaid. We are witnessing the production of ancestry, which should ensure its relevance. Analyzing the legitimation process shows old but still ever present logic of power, reactivated by an electoral national context. This article finally focuses on the way national consciousness expresses itself through ancestralization.
\end{abstract}

Keywords: ancestrality, ritual, politic, kinship, democracy, royalty, Madagascar, mermaid. 
$\mathrm{E}$

ntre le 21 octobre 2001 et le 21 janvier 2002, un cercueil a traversé le Sud de Madagascar d'est en ouest, depuis les environs de Tôlanaro (Fort-Dauphin) jusqu'à Toliara (Tuléar) ( $c f$. carte). Des centaines de villages se sont fait passer un "AncêtreSacré » (Razamasy), en un relais au cours duquel l'illustre défunt changea plusieurs fois d'identité. Parmi d'autres cas ${ }^{1}$, cet article présente cet exemple de créativité rituelle populaire comme lieu d'expérimentation d'une identité nationale partagée : un relais funéraire, dont l'observation permet d'assister à la création collective et trans-ethnique d'un ancêtre. Où l'ancestralité, critère de différenciation dans une société extrêmement hiérarchisée, est revisitée pour tenter de reconstruire un collectif à plus grande échelle. Ce rituel inédit - idée pour le moins paradoxale - va produire progressivement sa propre pertinence. Le processus de légitimation alors mis en évidence dévoile du même coup l'affirmation d'une identité nationale au travers d'un cadre de l'expérience qui combine patrimoine rituel et actualité politique.

\section{La procession d'une sirène assassinée}

Séjournant dans la ville de Toliara au moment des faits, j'ai pris connaissance de ce rituel inhabituel au moment où il prenait fin. Une rumeur, tout d'abord, a attiré mon attention. En croisant les versions, elle peut se résumer ainsi. À Fort-Dauphin, un pêcheur a pris une sirène dans ses filets, puis l'a ramenée à terre. Au village, la sirène est intriguée par l'activité de quelques hommes qui préparent du rhum. Elle demande à goûter, et boit trois bouteilles sans aucune ivresse. Apeuré, un des hommes frappe mortellement l'étrange créature qui, agonisante, maudit ses assassins puis donne ses dernières volontés : elle veut être enterrée au village d'Ankilibe près de Tuléar, où on la mènera à pied, en se relayant à chaque village, et ceux qui voudront lui demander bénédiction poseront la main droite sur elle.

Alors qu'à Tuléar la rumeur est à son comble, elle devient réalité : un petit cercueil est apporté aux notables d'un village situé à quelques kilomètres sur la route principale. S'enchaîne alors une séquence rituelle inédite, dont on peut distinguer cinq moments : (1) cortège (2) accueil (3) prières (4) veillée (5) ostension. L'objet matériel du culte est une caisse de moins d'un mètre entourée d'étoffes maintenues par un cordage et un drapeau malgache. Un objet relativement familier à Madagascar.

Les villageois croient que ce cercueil contient la dépouille d'une ampelamananisa, une "femme-avec-des-ouïes ». Cet être issu des fonds marins est en outre capable de contrôler les éléments, tels que l'orage et la pluie, ce qui représente un immense pouvoir dans une région semi-aride, régulièrement touchée par le manque d'eau et la famine. Il est masy, " sacré », et cet adjectif exprime à la fois la force efficace (hasy) que possèdent intensément les ancêtres, les souverains, les génies, et l'éminent statut que l'on confère à ces êtres.

1) Le cortège est composé de trois porteurs faisant partie du village précédent, qui resteront au village hôte jusqu'au prochain départ du Razamasy, les deux villages se rencontrent donc à mi-chemin. Le cortège obéit rigoureusement à une série d'interdits

1 Dans ma thèse, j'ai comparé ce relais funéraire, un culte antisorcellerie (Nord-Est, 1975-1983) et un culte de possession (ville de Toliara, années 1990 et 2000), (Mouzard 2011). 
qui sont censés avoir été énoncés par l'ancêtre lui-même, tels que sauter un village, arriver la nuit, utiliser un véhicule, porter sur l'épaule gauche, passer devant le Razamasy, etc. Le rituel se déroule à la manière d'un relais. Il n'y a pas d'officiant attitré, mais au contraire une prise en charge autonome, par chaque village successif, de l'ancêtre sacré.

2) Les porteurs, qualifiés de même qu'à un enterrement de tsimahaivelo, litt. " ceux-qui-ne-connaissent-pas-les-vivants » veillent à ce que les interdits soient respectés et indiquent le déroulement du rituel au village hôte. Ils commencent par déposer le Razamasy à l'est du tronc de l'arbre de la place principale du village (le tamarinier apparaît souvent sur la place principale d'un village en tant qu'entité liée aux ancêtres et aux esprits du sol). Tous les villageois déjà prévenus sont réunis dans un demi-cercle situé à l'ouest du tronc qui en forme le centre. Au sud, on trouve l'autorité administrative villageoise et le chef du lignage fondateur. La configuration spatiale est formée selon l'axe des actes rituels, orienté est-ouest, l'est marquant l'ancestralité (et la primogéniture), et selon l'axe de la vie sociale, orienté sud-nord, le sud pointant parfois dans cette région la direction du pouvoir, à l'inverse du reste de Madagascar.

Les tsimahaivelo du village précédent montrent aux notables des procès-verbaux officiels émanant des autorités étatiques de centaines de villages, et attestant que les coutumes ont été respectées et reproduites à chaque fois. Ils tiennent un discours explicatif quant aux formalités rituelles : il faut d'abord désigner trois nouveaux tsimahaivelo, puis les villageois doivent se cotiser pour couvrir les frais des produits nécessaires à la veillée : du riz, une chèvre à sacrifier, du rhum pour la libation et la consommation des porteurs, qui reçoivent aussi un don en argent. Le chef de lignage effectue ensuite une libation et une invocation adressées au Razamasy avant de procéder au sacrifice. Le sang est aspergé aux quatre points cardinaux, puis dans la direction que va suivre le Razamasy.

3) Les villageois se mettent alors en file dans l'axe et la direction de l'est. Ils doivent se soumettre aux interdits suivants : ne pas porter de chaussures, de chapeau, ne pas poser la main gauche sur le cercueil. Il est interdit à quiconque de stationner ou de traverser la zone située à l'est de l'espace de prière. En y pénétrant, les priants s'accroupissent, formant ainsi une file jusqu'au cercueil. Les tsimahaivelo indiquent aux priants comment ils doivent s'y prendre. Lorsque plus personne ne désire prier, les tsimahaivelo procèdent au comptage de la somme des offrandes recueillies dans une boite en carton. Ils font des petits paquets d'argent, qu'ils placent ensuite dans le cercueil, sous les linceuls.

Après la séance de prières, les notables sont face à deux alternatives : ou bien, si l'heure le permet, faire en sorte que le Razamasy arrive au prochain village avant la nuit, ou bien organiser une veillée, ce qui implique une deuxième dépense (famaha).

4) Le Razamasy est veillé comme on a l'habitude de veiller un mort. Grâce à un dispositif particulier, c'est le Razamasy lui-même qui couvre les dépenses et décide du montant accordé. Lorsque les habitants d'un village s'apprêtent à organiser une veillée, ils annoncent au Razamasy par l'intermédiaire des tsimahaivelo, la somme qu'ils jugent nécessaire pour couvrir les dépenses de la veillée. Ceux-ci plongent alors la main chacun leur tour sous les linceuls du cercueil et en retirent chacun une seule poignée de monnaie. Le montant prélevé dans le cercueil 'à l'aveugle' laisse croire 
qu'il est déterminé par l'Ancêtre-sacré lui-même, qui ne laisse prendre aux mains des tsimahaivelo que ce qu'il veut.

5) L'ostension s'effectue à l'aube de la veillée. Elle est précédée d'une libation et d'une invocation, effectuées par le hazomanga. Le contenu du cercueil n'est absolument pas manipulé. Une fois les linceuls écartés, tout est une affaire de regard. Ce que l'on voit alors est l'accumulation des offrandes, pièces de monnaie et billets de banque, et de petits papiers froissés en boule, desquels émergent indistinctement de courts ossements, un petit crâne, et une toison noire. Avant le départ du Razamasy pour le prochain village le hazomanga procède à une invocation semblable à celle effectuée à son arrivée. Le président du Fokontany rédige un procès-verbal mentionnant date et lieu de passage du Razamasy.

L'ultime étape de ce relais accompli sur environ 800 kilomètres devait être Ankilibe, terre ancestrale du Razamasy, selon la légende. Mais les funérailles n'ont pas été menées à terme, puisque des agents de l'État les ont réprimées. En outre, des universitaires sont intervenus pour effectuer un examen de la dépouille : il fut établi qu'il s'agissait de celle d'un aye-aye (Daubentonia madagascariensis). À l'issue de cette répression, la dépouille (délestée au passage de la somme des offrandes) aboutit au « musée régional de l'université de Tuléar ».

\section{L' origine du rituel : les funérailles du aye-aye en pays tanosy $\left(1^{\text {ère }}\right.$ phase $)$}

J'ai donc poursuivi la recherche au petit musée, où des priants continuaient à demander bénédiction. Les boulettes de papier que contenait le cercueil se sont avérées être des procès-verbaux dressés à chaque village. Après défroissage transcription et traduction des cent trente-cinq textes lisibles, l'élaboration d'une base de données chronologique avec date, lieu et désignation a permis de reconstituer sur une carte la trajectoire de la « procession ». Elle commence au Sud-Est, tout près de Fort-Dauphin, puis traverse trois ensembles géographiques : chaînes de montagne anosyennes, pénéplaine tandroy, puis axe routier de la plaine côtière mahafaly qui mène jusqu'à Tuléar.

L'origine en est le village d'Andramaka, situé à une dizaine de kilomètres de la ville de Fort-Dauphin, au Sud-Est de Madagascar, comme nous l'apprend le premier procèsverbal, daté du 24 octobre 2001, et dont voici la traduction (procès-verbal ci-contre).

Ce procès-verbal devait être contresigné à chaque nouveau village. Quand la place vint à manquer sur la feuille de format $\mathrm{A} 4$, on commença à rédiger de courts textes sur des petits bouts de papiers mentionnant date, lieu et passage d'un corps.

À Andramaka, des témoignages ont été recueillis. En croisant les versions, on aboutit à la reconstitution suivante : la journée du 21 octobre, un aye-aye a été aperçu par des enfants sur le toit d'une maison du village d'Andramaka. Surpris par cet animal qu'ils ne connaissaient pas $^{2}$ ces enfants ont fini par le tuer. Des anciens ont alors reconnu le hay-hay (nom tanosy, litt. «bien-connu »), et ont réactivé une coutume prête à sombrer dans l'oubli.

2 Il faut attendre jusqu'à 48 heures pour pouvoir observer le aye-aye dans son milieu naturel. Si l'on a pu penser l'espèce disparue, elle est en tout cas en voie de disparition. 
$=$ procès-verbal $=$

Aujourd'hui Lundi vingt et un Octobre année deux mille un aux environs de dix heures du soir on a vu à Amboanato, Fokontany d'Andramaka, Commune de Soanierana une bête morte dont on ignorait le nom, c'est-àdire : «DAUBENITONA MADAGASCARIENSIS, de son nom scientifique, ou bien qu'on appelle aussi : AYE-AYE (Hê-hê). Le fokonolona s'étant réuni pour l'examiner, les notables du village d'Andramaka ont dit que c'est une bête sacrée. Donc à sa mort on doit appliquer les coutumes ancestrales propres aux Malgaches. Sur le champ le Fokonolona a décidé d'envelopper cette bête de tissus blancs et a désigné des personnes pour la veiller exactement comme un mort. Beaucoup de gens y avaient aussi déjà déposé des offrandes. Le fokonolona a aussi décidé de faire une veillée funèbre d'un soir. Après cela on désignera des personnes pour le porter et le conduire jusqu' à un autre endroit, et puis les accompagnateurs reviendront. Les offrandes recueillies seront utilisées pour la veillée.

Comme le veut la coutume ancestrale, selon la tradition, ce défunt sera conduit de commune en commune.

MINISTÈRE DE LA DECENTRALISATION, DU BUDGET, ET DE LA PROMOTION DES PROVINCES AUTONOMES

FIVONDRONANA DE TOLAGNARO

commune de Soanierana.

« autorisation est donnée aux représentants du fokonolona de conduire le corps à un autre endroit. »

Fait à Soanierana le 24 octobre 2001. 
Si le aye-aye est souvent considéré comme un sorcier sur la côte orientale de Madagascar, on trouve dans le Sud-Est des représentations inverses, d'après lesquelles l'animal est considéré comme un ancêtre. En outre, cet être avec qui il est possible de lier amitié fait parfois don de son « oreiller », objet immensément précieux puisqu'il assurera à son détenteur une prospérité sans faille. Pour toutes ces raisons, on réserve dans cette région au aye-aye des funérailles spécifiques. Laissons M. Wilson, ancien d'Andramaka nous les décrire :

On l'amène dans des villages successifs, on le porte sur les épaules et on organise des veillées. On tue des boufs si on en a, on achète du rhum. Il y a des " mavo-kasoaty », ce sont des vieilles et elles sont chargées d'assurer la garde des morts. [...] Le matin suivant, on l'amène à un autre village. Chaque village où il est amené organise des veillées et ce jusqu'à ce qu'il ne reste plus que les os. Et un dernier village l'enterre. [...] Pas dans nos tombeaux. On lui construit un tombeau à part. Le jour où on l'enterre, on organise des fêtes, danses, luttes, combats de taureaux et on tue de gros boufs. Tout ce que l'on fait pour les funérailles, on le fait aussi pour le hay-hay. De grandes fortunes sont dépensées. Mais c'est celui sur qui est tombé l'Oreiller-du-Hay-hay qui fera fortune. Tous les villages dans lesquels il est passé, eh bien, c'est en quelque sorte comme la mort d'un roi, ils ne font que les festivités, ils ne reçoivent pas de bénédictions, c'est comme quand on fait les festivités pour les funérailles d'un roi. Un roi quand il est mort, ce sont les esclaves qui en profitent. Tandis que ceux qui prennent part aux festivités, ils boivent. Quoi qu'il en soit, il faut qu'ils reçoivent des boissons alcoolisées. [...] C'est la communauté villageoise qui désigne des porteurs (pour amener la dépouille du aye-aye au village suivant).

Après avoir sacrifié un bœuf afin de conjurer leur faute, la famille des tueurs du ayeaye a donc fabriqué un cercueil, acheté des linceuls, et organisé une veillée funèbre. Puis deux jeunes hommes ont porté à l'épaule la dépouille au village suivant, où a été rédigé le procès-verbal traduit plus haut.

Ce document administratif, à l'en-tête du ministère de la Décentralisation, du Budget, et de la Promotion des Provinces autonomes a un objectif patrimonial et juridique : expliquer une coutume qui n'est plus connue que de quelques anciens, et, comme il est courant, de légaliser le transport du corps.

\section{Transformations du rituel : variation sur le thème de l'ancestralité (phase de transition)}

Alors que le cadavre se réduit déjà à ses os, il n'est toujours pas enterré comme le prescrit la coutume, ce qu'atteste un procès-verbal datant du $1^{\text {er }}$ décembre 2001 (mais pas son écriture administrative). De plus, le même procès-verbal ne désigne plus la dépouille comme celle d'un hahay, mais de « kalanary ». Or ce n'est pas la première fois que change la désignation, ni la destination, puisque le 22 novembre 2001, on écrit que « cette bête a dit qu'elle voulait être enterrée à Tananarive ». La coutume est en train de se transformer.

Au-delà des montagnes de l'Anosy les caractéristiques physiques du milieu de vie repoussent le 'Daubentonia' madagascariensis, qui n'affectionne que les forêts toujours vertes du littoral Est. Les habitants de cette zone, dans laquelle on rencontre 
principalement les ethnonymes Tandroy ou Bara, ne connaissent pas pour la plupart ce rare lémurien, et a fortiori ne savent le reconnaître à son squelette. Le procès-verbal explicatif, qui aurait pu garantir une stabilité, a disparu sous la masse des suivants, car à chaque village une copie est chiffonnée et placée dans le cercueil. L'origine du rituel est devenue hors d'accès, et sa forme « en relais » engage sur cette base une chaîne de transmission qui subit de nombreuses distorsions.

Les procès-verbaux nous informent que dans cette phase de transition, qui va durer environ un mois et demi, soit plus d'un tiers de la durée de la procession, le nom et la nature de l'être dont le cadavre est en circulation va constamment changer. On trouve jusqu'à sept noms différents, qui ne se succèdent pas de manière linéaire, mais disparaissent et réapparaissent au long de la trajectoire erratique du petit cercueil dans la plaine tandroy. Les voici : Hahay ; Mandakahay; Lakahay; Biby omby ; kalay; Kalanary; et le nom scientifique ('Daubentonia' madagascariensis) diversement réinterprété. Ces noms sont associés sans grande régularité à au moins six natures différentes : biby (« bête, animal surtout sauvage ou être extraordinaire d'une nature supérieure »; razana (《 ancêtre »); biby razana ; javatra (« chose »); taolana (« ossements »); zanahary (« divinité »); tianake andriagahary/zanak Andriamanitra (« enfant de Dieu »).

À partir de la fin décembre commence une nouvelle phase de stabilité décrite plus haut. À partir de ce moment, il s'agira sur les procès-verbaux d'un razana, un " ancêtre », comme l'atteste le dernier procès-verbal retrouvé. Sur ce dernier, il est précisé que cet ancêtre vient de Fort-Dauphin et a pour destination Ankilibe (un village du littoral près de Tuléar). La « procession » emprunte à ce moment la RN10, qui relie l'agglomération de Tuléar au Sud de la province du même nom. Cet axe routier est incessamment parcouru dans les deux sens par des véhicules de transport collectif, bondés de voyageurs qui pour la plupart pratiquent un commerce individuel entre ville et campagne. La fréquence des arrêts de ces «taxis-brousse » tout au long de leur long itinéraire produit une intense et rapide circulation d'informations. Après ses circonvolutions rurales, ce nouveau milieu de vie garantit au rituel une stabilité sémantique jusqu'à sa fin. Le cercueil est désormais précédé d'un récit qui lui donne sens : celui de la sirène assassinée.

\section{D'étranges funérailles}

Tout débute donc avec la mort d'un prosimien présentant des caractéristiques morphologiques et comportementales particulièrement originales, prise en charge par une coutume rare lui conférant un statut ontologique élevé. Ce rituel funéraire réservé au aye-aye dans le Sud-Est revêt aussi une certaine originalité : pas d'officiants fixes, mais un relais autour de la dépouille. Chaque village (fokontany) traversé va prendre en charge le traitement du cadavre en changeant ses linceuls imprégnés de sanies qu'il jettera à la rivière, jusqu'au complet décharnement du corps. Ces deux caractéristiques se retrouvent dans l'Ikongo mais aussi en Isandra pour les rois et nobles le Betsileo (Razafintsalama 1983). Environ $400 \mathrm{~km}$ plus au nord, les anciens « rois » tañala « jetaient les sanies dans la Sandrananta ou d'autres rivières, en des endroits où l'eau est profonde et noire », et « par suite de la durée prolongée des funérailles - du moins pour les olombe [notables] -, on ne portait les corps au tombeau qu'après décomposition » (Beaujard 1983 : 462, 464). La première pratique s'expliquerait par une parenté des 
rois avec les esprits des eaux, et la seconde par une volonté de ne pas souiller les anciens corps du tombeau avec les nouveaux. Cette double opération rituelle dénote donc en tout cas, au moins sur le plan historiographique, un « statut » élevé conféré au hahay, que confirment les croyances locales et leur écriture. Chez les Sakalava, le rite mortuaire réservé aux rois implique également une décarnification surveillée, ne laissant à inhumer que les ossements (Decary 1962 : 237).

En revanche, l'acheminement du mort à sa dernière demeure ne se déroule pas à strictement parler en relais ni chez les Tañala ni nulle part ailleurs. Le choix de la destination finale est fixé, sinon par testament, généralement par application circonstanciée de la règle du " partage des morts ». Mais le trajet du lieu du décès jusqu'au tombeau est marqué par des haltes, car des parents négocient (ou imposent) le passage de leur ancêtre par leur propre village, où il sera placé dans la tranobe, la maison collective appartenant à une lignée. Les parents se livrent ainsi à une « bataille pour le mort » (ady faty), qui lui fait décrire jusqu'à son inhumation une trajectoire faite de tous les villages ayant réussi à obtenir son détournement chez eux afin de l'honorer, mais aussi de s'honorer eux-mêmes, et d'affirmer un certain prestige. Beaujard souligne que « ces luttes théâtrales ne doivent pas être considérées comme étant en contradiction avec la recherche d'une cohésion sociale » (Ibid. : 470), et insiste sur la fonction unifiante des funérailles, à travers lesquelles se manifestent le respect de la hiérarchie et la réelle volonté de dépasser les conflits.

De manière générale à Madagascar, les funérailles s'imposent comme les événements dans lesquels se jouent et se construisent l'identité et l'ordre social. Différentes études ont en effet bien montré comment les Sakalava (Feeley-Harnik 1991), Merina (Bloch 1971 ; Larson 1997), Betsimisaraka et Karembola (Cole et Middleton 2001), Antakaraña (Berger 2006), ont su transformer leurs pratiques funéraires en vue de préserver leur identité et la reproduction de l'ordre social en réaction à la pénétration coloniale. Le rituel des funérailles du aye-aye se présente ainsi comme un cas particulier de l'institution centrale de la société malgache. Seulement, l'arrière-plan rituel a beau être de toute importance, la coutume des funérailles du aye-aye, pas plus que l'être lui-même, ne sont connus au sortir de l'Anosy. On peut donc se demander pour quelles raisons tant de gens vont accepter d'accomplir le relais.

\section{Une trajectoire historiquement orientée}

Dans le premier village d'Andramaka, où le aye-aye a été tué par des enfants, des anciens issus du lignage fondateur exigent le sacrifice d'un zébu pour réparation. Puis le relais est lancé. Au second village, le cadavre est réceptionné par le maire, qui accepte cette coutume qu'il ne connaît pas, dans la mesure où, selon ses dires, elle appartient au patrimoine culturel de son pays. Il sait d'autre part, grâce aux campagnes de sensibilisation du WWF, que le aye-aye est une espèce protégée, qu'elle appartient au patrimoine de la biodiversité mondiale, et que sa circulation pourrait porter préjudice. Aussi, pour que ce patrimoine rituel ne devienne pas illégal tout autant que pour transmettre son sens et son déroulé, il dresse un procès-verbal officiel.

$\mathrm{Au}$ troisième village la situation est encore différente. La dépouille du lémurien est désormais accompagnée d'un document officiel à en-tête du tout récent ministère de la Décentralisation, du Budget, et de la Promotion des Provinces autonomes sur lequel on 
peut lire : « les notables du village d'Andramaka ont dit que c'est une bête qui a du sacré. Donc à sa mort on doit appliquer les coutumes ancestrales propres aux Malgaches ». Dès lors se trouvent enchâssées deux structures d'autorité : celle des notables du prestigieux village d'Andramaka dans celle de l'État. C'est donc très probablement cette structure d'autorité qui permet d'expliquer l'acceptation de la coutume par les villages suivants. Cependant, arrivé au village de Sampona, le cercueil fait demi-tour car ses notables refusent eux de rendre un culte à un animal qu'il ne connaissent même pas, justifiant cette abstention au nom de leur identité ethnique (tandroy). Ni l'autorité des notables d'Andramaka, ni celle de l'État, exprimées à travers le procès-verbal ne sont donc suffisamment importantes pour que ces Tandroy acceptent le rituel.

Cependant, alors qu'un mois plus tard le cercueil franchit les montagnes de l'Anosy, échouant à des villageois qui pourraient tout autant que ceux de Sampona faire valoir leur identité pour le repousser, le relais se perpétue. Pour expliquer cela, on peut tout d'abord avancer un fait historique de peuplement. Les conquêtes merina engagées par Radama $I^{\text {er }}$ au début du $19^{\text {ème }}$ siècle laissèrent tout le Sud du pays indépendant, à l'exception de l'Anosy, soumis en 1825. Après l'échec d'une première révolte tanosy, une partie de la population a pris la fuite vers l'Ouest, en contournant le pays antandroy par le nord. En 1840 une deuxième révolte est suivie d'une répression par les agents de Ranavalona I re, provoquant en 1845 une migration bien plus massive de 30000 individus. Ils se seraient frayés un passage dans la zone inoccupée à l'ouest de Tsivory jusqu'au moyen Onilahy, où ils devinrent les vassaux du souverain mahafaly de la région. Aux migrations collectives des siècles précédents succèdent sous la colonisation des migrations beaucoup plus individualisées, mais qui continuent à alimenter l'implantation d'originaires du Sud-Est du haut bassin de la Mandrare jusqu'à la région comprise entre l'Onilahy et le Fiherena (Deschamps 1959 : 263). Ainsi, les populations actuelles de la région de la haute Mandrare (vers Tsivory) conservent une mémoire sociale de leur origine, puisqu'elles « affirment venir du Sud, et plus précisément du Sud-Est : région de Ranopiso-Fort-Dauphin » (Radimilahy 1981 : 103 ; Ranopiso est situé à quelques kilomètres d'Andramaka). Ce trajet migratoire est d'ailleurs bien antérieur au $19^{\text {ème }}$ siècle, puisque le peuplement global du Sud de la Grande Île dans la deuxième moitié du $16^{\text {ème }}$ siècle est l'œuvre d'individus qui se sont détachés du SudEst pour aller fonder des royaumes dans l'intérieur (Deschamps 1960). Le fait que le Razamasy soit repoussé au sud-ouest du pays tanosy alors que la « contagion » opère au nord-ouest pourrait donc s'expliquer par une continuité géoculturelle, ou tout au moins la conscience historique d'être originaire du Sud-Est, tout comme l'ancêtre en circulation. Cette hypothèse explicative paraît se confirmer quand on s'aperçoit que la frontière ethnique mise en évidence par la trajectoire du relais au niveau de la basse Mandrare (au village de Sampona qui n'a pas reconnu l'ancestralité du défunt) a été repérée au $17^{\text {ème }}$ siècle (Flacourt 2007 : 147). Le début de la phase de transition représente donc une plongée en profondeur dans l'histoire du Sud de Madagascar, et du pays en général. La trajectoire du squelette, qui recoupe celle du peuplement par les migrations des anciennes dynasties, avec une origine sud-orientale qui correspondrait au foyer de toutes les monarchies malgaches, assure une continuité des ressources symboliques $\mathrm{du}$ rituel. Les acteurs à ce moment ne savent pas nécessairement que la mort a eu lieu un mois avant que le corps ne leur parvienne. Ils peuvent bien au contraire imaginer 
que ces ossements en désordre contenus dans une petite boite proviennent d'un passé lointain, si lointain qu'il appartient à ce que nous nommons mythologie.

\section{Écriture étatique, écrits populaires}

Ce facteur historique ne paraît donc pas négligeable, cependant, il ne peut sans doute pas expliquer à lui seul la différence de comportement constatée aux deux endroits du relais où celui-ci quitte l'Anosy. En effet, la situation diffère à d'autres égards. Vingt jours se sont écoulés depuis le refus de Sampona, quand le corps du Daubentonia se retire de la région de l'Anosy pour commencer sa sinueuse descente entre les cuestas de la Mandrare. D'une part on peut estimer que l'animal est quasiment inconnu dans cette région. D'autre part son cadavre est à moitié décomposé, puisqu'au bout de quarante jours de relais ne subsistent que les os et la toison ( $\mathrm{Pv} 91$ et suivants, sur lesquels on fait mention «d'ossements ", taolana). Enfin plus d'une cinquantaine de procès-verbaux se sont accumulés dans la caisse, vraisemblablement sous le corps, ou aux extrémités, ce qui représente près de la moitié du volume du cadavre. Une phase de transition s'amorce alors, caractérisée par l'alternance des désignations. Cette phase se fonde sur une méconnaissance de l'animal, ce qui n'est pas le cas à Sampona, où l'on était assuré d'avoir affaire à un lémurien. Or cette incertitude ne fait qu'accroître le symbolisme de la chose, et l'on prendra plus difficilement position contre une dépouille ancestrale dont on ignore la nature exacte, que l'on ne se défendra de rendre un culte à un animal.

De plus, la masse des procès-verbaux exprime le message selon lequel la dépouille est originaire du Sud-Est, mais matérialise aussi la participation d'un nombre désormais conséquent de communautés villageoises. Ce nouvel input est donc à mettre en relation avec l'arrière-plan historique de la trajectoire qu'il contribue à évoquer, mais aussi à prendre en compte afin de saisir l'évolution du mécanisme de légitimité. Ce qu'expriment les procès-verbaux aux yeux des participants est tout autant la légitimité étatique à travers la pratique du rapport protocolaire, qu'une légitimité populaire. Naturellement, plus le relais progresse, plus le cercueil - dans lequel on trouve la preuve matérielle de cette accumulation - a parcouru de villages et traversé d'espaces sociaux différents, et plus cette légitimité s'accroît. Ainsi les notables d'Ankilibe, en bout de chaîne, insistent-ils sur ce point pour justifier leur adhésion par opposition à l'attitude répressive des agents de l'État. Autrement dit, en considérant la série du rituel et pas seulement sa séquence, on observe une rétroaction du phénomène sur le phénomène lui-même. Ses effets peuvent ainsi en expliquer le développement et la persistance. Ce phénomène de « masse critique » des boulettes de procès-verbaux participe donc à la légitimation d'un rituel inédit, ajoutant à une légitimité étatique et historique une reconnaissance populaire qui rend la chose acceptable et, pourrait-on dire « chosepublique ». De plus, nul doute que la méconnaissance de l'animal et des faits précis à l'origine de sa mort et de ses funérailles n'accroissent l'adhésion au rituel si tant est que « l'action sociale efficace repose sur l'effacement des mécanismes cognitifs qui la structurent » (Descola 2005 : 167). Mais d'autres facteurs sont à prendre en compte dans ce procès de légitimation. 


\section{Les urnes et le cercueil}

La phase de transition s'amorce en même temps que la campagne présidentielle. À la mi-novembre, le petit squelette descend des montagnes de l'Anosy pour commencer son parcours erratique, cependant qu'à Tananarive les deux grands candidats à la présidence descendent dans les stades pour de grands meetings. Trois jours avant cette date, on écrit justement que le Razamasy « a dit qu'il voulait être enterré à Tananarive ». Coïncidence ? C'est pendant la quinzaine de jours qui précède le premier scrutin présidentiel que la dépouille sacrée est qualifiée de "divinité en provenance de l'Anosy », puis « d'enfant-de-Dieu » (en dialecte du Sud, puis des Hautes-Terres). Comment interpréter cette volonté populaire d'emmener un " enfant de Dieu » à la capitale, au moment des élections présidentielles? Pourrait-il s'agir d'autre chose que d'une simple coïncidence?

Il semble bien que des conceptions politiques partagées par la plupart des anciens royaumes puissent fortement réduire l'arbitraire de cette congruence, dans la mesure où elles restent prégnantes dans la société actuelle, ce que d'importants ouvrages pour la compréhension de Madagascar mettent en avant. Ainsi dans l'introduction aux Souverains de Madagascar, l'histoire royale et ses résurgences contemporaines, F. Raison-Jourde (1983 : 17) écrit-elle : « Force est de reconnaître que si les dynasties sont éteintes, les représentations liées à la royauté (celle des hiérarchies sociales, celle de la légitimité) sont présentes non seulement dans la conscience populaire, mais dans celle des principaux acteurs politiques ». Ainsi Ottino (1998 : 581-91) conclut-il son opus sur les Champs de l'ancestralité en termes de " pesanteurs du passé », et tout récemment D. Galibert (2009) termine son étude de l'État post-colonial par un chapitre intitulé « la mémoire matricielle des monarchies ». P. Ottino (1983) fait remonter au moins au $13^{\text {ème }}$ siècle l'arrivée et l'application à Madagascar de symboliques indonésiennes du pouvoir, identifiées dans le cycle mythique des Andriambahoaka de Matacassi (Zafiraminia - le village d'Andramaka où le aye-aye a été trouvé est régionalement connu comme zafiraminia) et d'Imerina (14 ${ }^{\text {ème }}-17^{\text {ème }} \mathrm{s}$.), dont on trouve des remaniements dans toute la Grande Île. Les Andriambahoaka - qui est le nom donné au roi dans un certain nombre de mythes de souveraineté des Hautes Terres et des côtes malgaches, mais aussi le titre royal jadis en usage dans le Sud-Est de Madagascar - sont des « souverains universels » (Andriambahoaka) « au Centre de la Terre »(afovoan'ny tany). Dans différents systèmes monarchiques, le pouvoir de ces rois-dieux est mis en scène architecturalement et aussi dramatiquement par des danses sacrées, circumambulations et pèlerinages rituels marquant par un parcours des quatre points cardinaux la prise de possession de l'univers. Le monarque tire sa puissance mystique de l'alliance d'un ancêtre direct avec une princesse sacrée, fille de Dieu. Or P. Ottino (1983) développe à propos de « l'ancienne succession dynastique malgache » la thèse qu'à chaque nouveau règne, cette vertu légitimante s'étant épuisée, elle doit être à nouveau puisée à sa source par l'intermédiaire des princesses sacrées en ligne utérine. On peut juger fortuit le rapprochement de cette logique indonésienne de décroissance de rang et de rotation du pouvoir, avec un rituel populaire de l'extrême Sud malgache, même en gardant à l'esprit que les gens concernés ont mémoire de leur origine sud-orientale, qui reste aussi la référence historique des monarchies malgaches. L'argument semble pourtant éclairant concernant l'irruption d'une fille divine en pleine 
élection présidentielle, d'autant plus que d'autres faits corroborent une interprétation en termes de conceptions politiques d'ancien régime.

Trois jours avant que les présidents de fokontany ne consignent officiellement qu'un « enfant de Dieu » est passé par chez eux, le procès-verbal est retrouvé ${ }^{3}$, et celui-ci mentionne clairement que la chose « a du hasina ». Or hasina est précisément - en malgache officiel et en merina, car les gens du Sud disent hasy - la vertu mystique détenue par le souverain, garante de la fertilité et de la fécondité du royaume, dont les principaux rituels monarchiques ont pour fonction d'assurer le renouvellement et la dispensation, en un mot, la transmission. Funérailles royales, intronisation, circoncision et bain royal apparaissent comme des manifestations majeures du hasina et, selon M. Bloch (1983), véhiculent un même message : « le pouvoir politique n'est qu'un aspect d'un pouvoir mystique distribué à la population à différents degrés ». Dans le bain royal (fandroana) en particulier, qui remplit la même fonction de régénérescence de la puissance du souverain que les bains des reliques sur la côte ouest, un don au roi, individuel et pécunier, a lieu. Ce don se nomme hasina, et Bloch propose de l'appeler « hasina type II » pour le distinguer de la «puissance mystique » " hasina type $I »$ distribuée à la population via le souverain. Le hasina (I), essence dispensant la fécondité et se déplaçant du supérieur aux inférieurs, s'oppose ainsi au hasina (II), reconnaissance par un don de la supériorité de statut. Dans les anciens royaumes du centre de Madagascar, ce don consistait en une pièce d'argent, dont la valeur symbolique tenait en partie à ce que celle-ci soit entière, quand la majorité des pièces avaient été découpées en quartiers afin de créer de plus petites valeurs d'échange. Ici encore, un parallèle peut être fait avec l'offrande au Razamasy, et l'interdiction d'offrir une somme d'argent en plusieurs pièces ou billets.

« Les monarchies font très généralement référence, pour situer leur origine dynastique, au Sud-Est de Madagascar, c'est-à-dire une zone côtière ou s'opérèrent des débarquements successifs de migrants, compliqués par des déplacements internes, générateurs d'alliance ou de conflits » note Raison-Jourde (1983 : 18) dans son introduction aux Souverains de Madagascar. Sur le procès-verbal initial, le aye-aye est décrit comme « une bête, mais une bête qui a du hasina ». Et le voici maintenant fille de Dieu venant du foyer des fanjakana lehibe, des royautés malgaches, et voulant se rendre à Tananarive, alors qu'à ce moment même, on renouvelle la tête du fanjakana malagasy, de l'État. S'agit-il de renouveler le hasina du chef de l'État, qui le 25 novembre fait campagne au stade de Mahamasina, « qui rend-sacré » là où jadis avaient lieu les rituels d'intronisation ? Notons que dans la région où le aye-aye est devenu une fille de dieu l'électorat du président D. Ratsiraka a quadruplé en cinq ans, poussée nettement remarquable par rapport au reste du pays (Razafindrakoto et Roubaud 2002 : 36). Notons aussi que le crâne de la dépouille est désormais entouré d'une étoffe rouge, qui est la couleur des souverains partout à Madagascar (Beaujard 1991). L'utilisation de deux termes spécifiques renforcent cette connotation politique du rituel dans sa deuxième phase. Premièrement, ce qui permet de porter la dépouille est appelé à ce moment-là filanzana, soit la chaise à porteur avec laquelle on véhiculait

3 Le même jour du 4 décembre 2001, on passe de la Kalanary (pv103) au 'Daubenitona' Madascariensis (pv104) reproduit avec la même réinterprétation graphique que sur le tout premier document tapé à la machine (pv1). 
les rois (puis les colons), objet mettant en scène le pouvoir donc, et sans solution de continuité entre monarchies et colonisation. Deuxièmement, les porteurs du cercueil sont appelés les voromahery, littéralement " oiseau-fort », symbole de la royauté, selon un héritage austronésien (Beaujard 1983 : 281-2), nom des troupes du fameux souverain Andrianapoinimerina, à l'origine du royaume d'Imerina dont l'hégémonie unifia la quasi-totalité de la Grande Île ${ }^{4}$.

\section{Modalisations monarchiques et démocratiques}

Comment peut-on rendre compte de cette co-occurrence entre rituel démocratique national et rituel politico-religieux régional ? Formulé en termes de cadre, le problème devient plus clair. L'apport de E. Goffman sur la cadre-analyse initiée par G. Bateson consiste à avoir mis en évidence la « vulnérabilité » des cadres humains de l'expérience : « la perception d'une séquence d'activité mobilise des règles ou prémisses d'un cadre primaire, social ou naturel et peut donner lieu à deux types de transformations : les modalisations et les fabrications » (Goffman 1991 : 242). Un cadre primaire «permet, dans une situation donnée, d'accorder du sens à tel ou tel de ses aspects lequel autrement serait dépourvu de signification » (Ibid. : 30). Par " mode », Goffman entend « un ensemble de conventions par lesquelles une activité donnée, déjà pourvue d'un sens par l'application d'un cadre primaire, se transforme en une autre activité qui prend la première pour modèle, mais que les participants considèrent comme sensiblement différente » (Ibid. : 52). Un cadre peut contenir plusieurs remodalisations, donc plusieurs "strates supplémentaires à l'activité », dont la strate externe, la frange « indique, indépendamment du degré de complexité des différentes strates, le statut de l'activité dans le monde réel » (Ibid. : 91).

Le phénomène étudié ici manifeste, au niveau de la série et plus particulièrement de la phase de transition couvrant la moitié de sa durée totale, de très fréquentes modalisations. La vieille coutume tanosy des funérailles du aye-aye consiste déjà en une modalisation d'un cadre primaire, dont on retrouve les traces par exemple dans les descriptions des funérailles royales des Zafirambo décrites par Beaujard. Le début de la phase de transition, marquée par la disparition du texte explicatif et la méconnaissance du aye-aye, consiste donc d'abord en une démodalisation : la frange du cadre a été retirée. Mais puisque le squelette n'est pas perçu comme étant celui d'un roi (sa nature extra-humaine demeurant une constante du relais), ce repli sur le cadre primaire des funérailles royales consiste plutôt en une modalisation des funérailles du aye-aye. Ce rite autour des ossements d'un être connu-inconnu originaire de l'Anosy sera ainsi vécu sous le mode monarchique, comme l'atteste l'apparition du foulard rouge et du terme filanzana.

Les procès-verbaux participent du cadre, et en tant que méta-communication, dans les termes de G. Bateson (1977), ils sont à la fois la séparation et le lien entre l'intérieur et l'extérieur du rituel. Leur message ainsi est double : d'une part « ceci est [tel être sacré] » et d'autre part « ceci est officiel », autrement dit accepté par le fanjakana, l'État. Cet écrit tisse donc un lien entre le rituel et la dimension étatique

4 On accède aux palais royaux de Tananarive en passant sous un arc surmonté d'un aigle de bronze, emblème de la royauté (donné par Napoléeon III à Ranavalona $1^{\text {ère}) . ~}$ 
de son environnement. Or cet ancrage « officiel » dans le monde quotidien survient au moment où celui-ci est précisément marqué par le renouvellement du chef de l'État. La frange officialisante du rituel le lie donc avec le rituel électoral. À ce moment a lieu justement une véritable course aux procès-verbaux des bureaux de vote, le Sud du pays étant un enjeu important qui pourrait contredire les $60 \%$ du challenger de Ratsiraka, selon la déclaration du ministère de l'Intérieur. Cette institution pilotée par Ratsiraka entreprend alors la collecte par télécommunications, quand Ravalomanana envoie des hélicoptères pour récupérer directement les urnes et les centraliser sur la capitale. Au même moment, pendant le dernier tiers de la phase de transition, les procès-verbaux sont désormais placés dans une pochette en plastique transparent qui accompagnera le cercueil jusqu'au bout (ces documents n'ont hélas pas été remis au musée). L'année 2001 est particulièrement riche en rituel démocratique, si l'on en juge son calendrier électoral : conseillers des provinces autonomes, sénatoriale, gouverneurs des provinces autonomes, présidentielles.

Un coup d'œil sur l'ensemble de la période succédant à ce que l'on a nommé « transition démocratique » montre d'ailleurs un rythme accru d'échéances électorales à répétition et de referendums constitutionnels dans la Grande Île en 1992, 1993, 1995, 1998, 1999, 2000, 2001, puis 2006, 2007 et 2008 (pas moins de vingt-quatre élections ont été organisées en 48 ans, soit une élection tous les deux ans, en moyenne). C'est évidemment à une crise de la légitimité que cette surenchère électoraliste renvoie, ce qui ne fait que renforcer l'interprétation du relais sacré proposée ici.

La troisième phase présente une nouvelle modalisation, où on notera la disparition de la connotation monarchique. L'étoffe rouge nouée autour du crâne n'est plus, mais en revanche le cercueil parcourant la route nationale est noué par un étendard malgache tricolore. Il semble bien que la dimension capitale ait cédé le pas à une dimension plus totale. Le squelette ne doit plus être porté à Tananarive, mais à Ankilibe, village vezo situé à quelques kilomètres du pôle régional de Tuléar, et considéré comme la « terre ancestrale » (tanindrazana) de l'Ancêtre. Cette particularisation ethnique semble assez mal s'accorder avec une dimension totalisante sur le plan identitaire, perçue dans le cours de la procession. Or les particularités de l'ethnicité retenue atténuent cette contradiction. Le référent ethnique vezo du Razamasy ne restreint que de manière très limitée sa portée globale, puisque n'importe quel malgache peut devenir vezo. S'il est ancêtre de Vezo, cet ancêtre est ainsi potentiellement celui de tous, dans la mesure où cette identité se fonde sur le dépassement de la filiation (Astuti 1995). De plus, les Vezo, à l'instar des Tsimihety (Wilson 1992), ont développé un ethos égalitaire rejetant en partie la hiérarchie, sinon toute forme de centralisation du pouvoir. Si sous le mode monarchique la divinité fut associée au centre national du pouvoir, la trajectoire d'une sirène nationale aboutit sur le littoral de ces Malgaches si peu territorialisés ${ }^{5}$, et la dernière libation est faite en direction de la mer.

L'attention à la rumeur permet ici (comme y invitent notamment $\mathrm{S}$. Ellis et G. Ter Haar 2004) de cerner un peu mieux les choses. Au cours de la troisième phase, bien en amont d'Ankilibe, un nom a circulé sur la route nationale 10 : celui de Larantsy, pêcheur légendaire considéré régionalement comme un " héros culturel ». Il est frappant que dans un contexte national de crise démocratique - au moment de la

5 Les Vezo ont été semi-nomades jusqu'à une époque récente. 
troisième phase, les manifestations qui contestent les premiers résultats ont commencé à Tananarive place du 13 mai 1972 - il s'agisse d'amener au tombeau d'un héros culturel une parente sirène, donc de renouveler son hasina. En définitif, ce serait lui, Larantsy, que tout ce processus rituel électif aurait nommé. Pourtant ce n'est pas à son tombeau que les notables d'Ankilibe avaient décidé de placer les ossements sacrés. Les notables, conscients et respectueux de l'importance sociale de l'ancêtre vénéré depuis Fort-Dauphin, mais divisés sur le fait d'inhumer la dépouille sacrée au tombeau ${ }^{6}$ choisissent d'utiliser la somme des offrandes pour construire un petit monument «sur le modèle du palais de la reine » (Manjakamiadana). Ce n'est pas ici le retour d'un mode « monarchique ", mais l'apparition d'un patrimoine architectural national, une façon de dire, en plaçant ce lieu de prière au bord de la route, que cet Ancêtre-Sacré est celui de tous les Malgaches. La production populaire d'un ancêtre de portée nationale rencontre ici la pratique commémorative du nationalisme officiel ${ }^{7}$.

\section{Conclusion}

Les champs de l'ancestralité à Madagascar de P. Ottino (1998) nous fait accéder à une compréhension ethnographiquement intime et théoriquement solide des relations de parenté de diverses sociétés de la Grande Île avant de nous mettre face à leur effondrement historique. En se basant sur une ethnographie remarquable constituée au cours des trente premières années suivant l'indépendance, cette étude pose ainsi dans sa conclusion la question de l'avenir des formes de sociabilité envisageables et les possibilités d'ouverture de la parenté et de l'ethnie, notamment sur un horizon national porté par l'État postcolonial.

Le relais funéraire de 2001 semble bien répondre à cet enjeu sociologique, dans la mesure où ce rituel crée une intersubjectivité trans-ethnique à caractère national. Le registre de l'ancestralité est dépassé par le haut, quand les acteurs mobilisent successivement des figures imaginaires partagées opérant une médiation générale. La trajectoire du mort reprend un mouvement géo-historique significatif et fédérateur. Ce scénario découle d'un écrit administratif, qui manifeste une "réflexivité dérivée » (Rozenberg 2011) à partir d'une coutume en voie de disparition, et lui confère une dimension officielle et nationale. La perte de cet explicatif entraîne ensuite une " réflexivité instrumentale » (Ibid.) et de multiples recadrages du rituel au cours du relais. Une modalisation à connotation monarchique en lien avec sa trajectoire géohistorique, précède une remodalisation par homologie de forme et analogie de sens avec son actualité politique. Sur fond de crise démocratique élitiste, la créativité rituelle populaire développe un contrepoint unitaire en revisitant l'ancestralité.

6 Le chef de lignage auquel appartient Larantsy nie sa parenté avec une sirène.

7 Voir Gallibert (2009 : 179-217), chapitre de son ouvrage dans lequel l'auteur étudie chronologiquement les dramaturgies officielles instituées depuis 1960 et les lieux de mémoire établis par l'État post-colonial. 


\section{Références citées}

Astuti, Rita, 1995. People of the Sea. Identity and Descent among the Vezo of Madagascar. Cambridge : Cambridge University Press.

BAteson, Gregory, 1977 [1955]. Vers une écologie de l'esprit. Paris : Seuil.

Beaujard, Philippe, 1983. Princes et paysans. Les Tañala de l'Ikongo. Un espace social du Sud-Est de Madagascar. Paris : L'Harmattan.

-, 1991. Mythe et société à Madagascar (Tañala de l'Ikongo). Le chasseur d'oiseau et la princesse du ciel. Paris : L'Harmattan.

Berger, Laurent, 2006. Les raisons de la colère des ancêtres Zafinifotsy (Ankaraña, Madagascar). L'Anthropologie au défi de la mondialisation. Thèse de doctorat d'anthropologie sociale et Ethnologie. Paris : EHESS.

Bцoch, Maurice, 1971. Placing the Dead. Tombs, Ancestral Villages and Kinship Organization in Madagascar. Londres-New-York : Seminar Press.

-, 1983. « La séparation du pouvoir et du rang comme processus d'évolution », in Fr. Raison-Jourde (éd.), Les souverains de Madagascar. L'histoire royale et ses résurgences contemporaines, pp. 265-298. Paris : Karthala.

Cole, Jennifer et Karen Middleton, 2001. « Rethinking Ancestors and Colonial Power in Madagascar », Africa, 71 (1), pp. 1-37.

DeCARY, Raymond, 1962. La mort et les coutumes funéraires à Madagascar. Paris : Maisonneuve.

Deschamps, Hubert, 1959. Les migrations intérieures passées et présentes à Madagascar. Paris : BergerLevrault.

-, 1960. Histoire de Madagascar. Paris : Berger-Levrault.

Descola, Philippe, 2005. Par-delà nature et culture. Paris : Gallimard.

Ellis, Stephen et Gerrie Ter HaAr, 2004. Worlds of Power, Religious Thought and Political Practice in Africa. New York : Oxford University Press.

Feeley-Harnik, Gillian, 1991. A Green Estate. Restoring Independance in Madagascar. Washington : Smithsonian Institution Press.

Flacourt DE, Étienne, 2007 [1650]. Histoire de la grande isle de Madagascar. Paris : Inalco-Karthala.

GALIBERT, Didier, 2009. Les gens de pouvoir à Madagascar. État postcolonial, légitimités et territoire (19562002). Paris : Karthala.

Goffman, Erving, 1991 [1974]. Les cadres de l'expérience. Paris : Editions de Minuit.

Handelman, Don, 2004. « Re-Framing Ritual », in J. Kreinath et al., The Dynamics of Changing Ritual, pp. 9-20. New York-Oxford : Peter Lang.

Larson, Pier, 2001. «Austronesian Mortuary Ritual in History. Transformations of Secondary Burial (famadihana) in Highland Madagascar », Ethnohistory, 48 (1-2), pp. 123-157.

LuPo, Pietro, 2003. «L'idée de sacré (hasina) et d'autorité dans la pensée religieuse traditionnelle », mémoire de 1'Académie Nationale Malgache, Série Lettres et Sciences humaines, 47, pp. 113-122.

MouzArD, Thomas, 2011. Territoire, trajectoire, réseau. Créativité rituelle populaire, identification et État postcolonial (Une triple étude de cas malgache). Thèse de doctorat en anthropologie sociale et etnologie. Paris : EHESS.

OtтіNo, Paul, 1983. « L'ancienne succession dynastique malgache (l'exemple merina) », in F. Raison-Jourde (éd.), Les souverains de Madagascar. L'histoire royale et ses résurgences contemporaines, pp. 223263. Paris : Karthala. 
-, 1998. Les champs de l'ancestralité à Madagascar. Paris : Karthala.

Radimilahy, Chantal, 1981. « Migrations anciennes dans l'Androy », Omaly sy Anio, 13-14, pp. 99-111.

RaISON-JOURDE, Françoise (dir.), 1983. Les souverains de Madagascar. L’histoire royale et ses résurgences contemporaines. Paris : Karthala.

RaZAFindrakoto, Mireille et François Roubaud, 2002. « Le scrutin présidentiel du 16 décembre $2001:$ les enjeux d'une élection contestée », Politique africaine, 86, pp. 18-45.

Razafintsalama, Adolphe, 1983. " Les funérailles royales en Isandra d'après les sources du $19^{\text {ème }}$ siècle », in F. Raison-Jourde (éds) : L'histoire royale et ses résurgences contemporaines, pp. 193-209. Paris : Karthala.

Rozenberg, Guillaume, 2011. « Magie du rituel, démon de la réflexivité », L’Homme, 198-199, pp. 277-299.

Wilson, Peter J., 1992. Freedom by Hair's Breadth : Tsimihety in Madagascar. Ann Arbor : University of Michigan Press. 


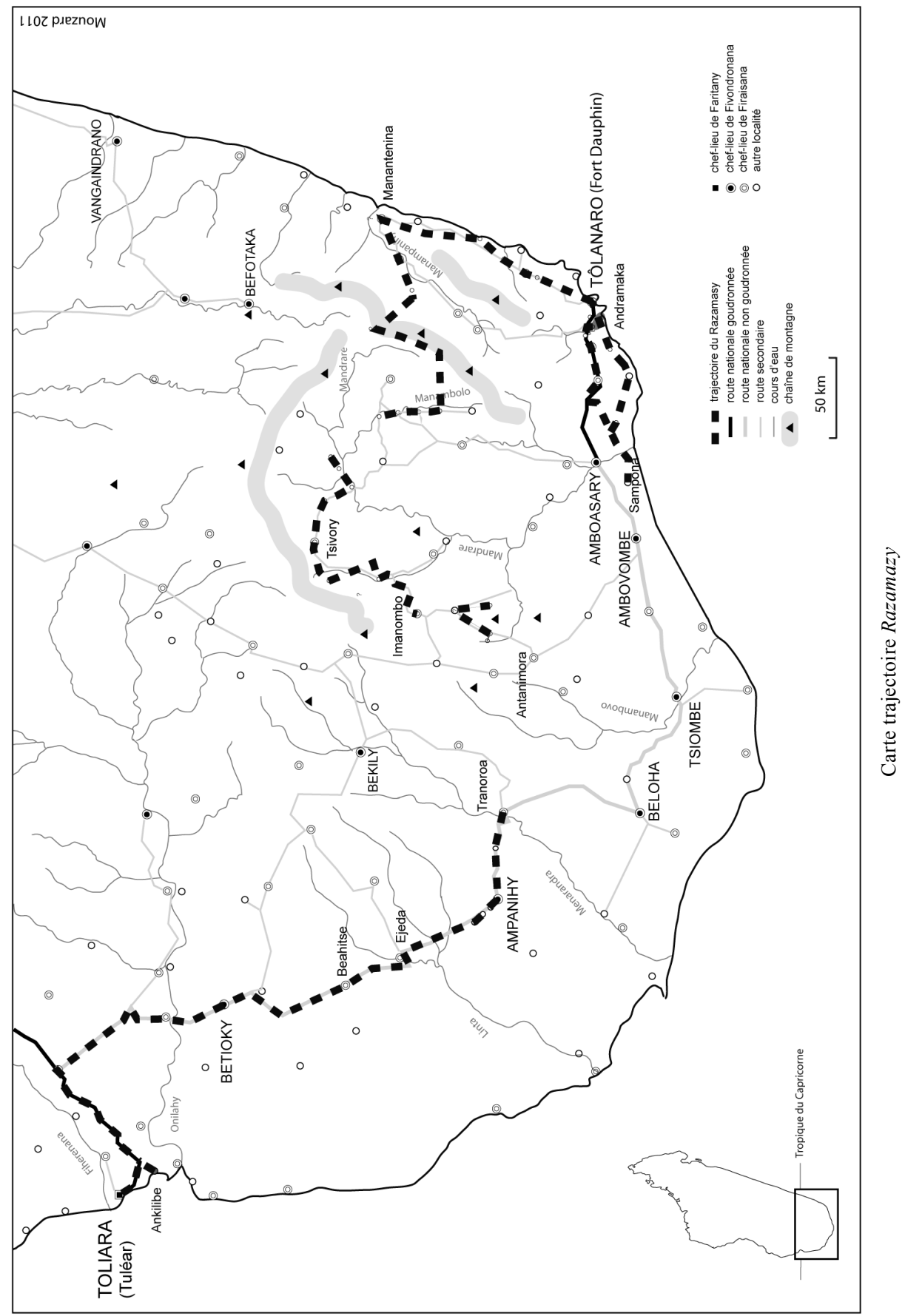

